\title{
Risk Factors of Non-Alcoholic Fatty Liver Disease in Bangladeshi Population
}

\author{
MOSTAFA KAMAL ${ }^{1}$, FAZAL KARIM², MD.ROBED AMIN ${ }^{3}$, MH SARDER ${ }^{4}$, KAK AZAD $^{-5}$
}

\begin{abstract}
:
Background: Non-alcoholic fatty liver disease (NAFLD) is becoming a public health problem with increasing incidence and it has been shown to be associated with diabetes, dyslipidemia, obesity and metabolic syndrome and ultimately puts more than half the world's population at risk of developing NAFLD/NASH/cirrhosis in the coming decades. The importance of detection of patients with NAFLD is to intervene the associated factors and avoid transformation to more severe forms of the disease.
\end{abstract}

Methods: An observational case control study was carried out at Medicine Department, Dhaka Medical College Hospital (DMCH), Dhaka, during the period of August, 201I to June, 2012. A total number of 100 consecutive patients and 100 healthy controls was approaches $\mathrm{DMCH}$ were enrolled in this study. Out of 100 NAFLD patients only 20 patients gave consent for liver biopsy to see the extent of liver damage.

Result: Less physical activity, Obesity, mean blood pressure, Diabetes, TG, TC were significantly $(P<0.05)$ higher in NAFLD. Metabolic syndrome was $64 \%$ in NAFLD. According to NASH Activity Scoring system definite NASH (score $>5$ ) was found in $9(45 \%)$ of the NAFLD group.

Conclusion: Significant proportion of NAFLD patient suffers from NASH which is alarming and they have potential for the development of advanced liver disease. So recognition of these risk factors in patients of NAFLD can help in early implementation of strategies that halt the progression of this disease.

Key words: NAFLD, NASH, CLD

\section{Introduction:}

NAFLD consists in the accumulation of fat vacuoles in the cytoplasm of hepatocytes and is characterized by the presence of hepatic lesions similar to those made by alcohol in subjects without significant alcohol consumption. This term was introduced by Ludwig in 1980 and covers a wide spectrum of hepatic lesions including simple fatty liver, steatohepatitis with necroinflammatory changes and a variable degree of fibrosis which may finally progress to liver cirrhosis and, even hepatocelluar carcinoma. However, there is little information on the clinical relevance of this disorder as a healthcare problem in the general population, since the studies published generally include a limited number of patients and the diagnosis is established on the basis of clear biochemical alterations and liver biopsy ${ }^{1,2}$. Many etiologic factors are associated with NAFLD and are classified as primary, related to factors that cause an increase in insulin resistance and the metabolic syndrome (obesity, type 2 diabetes, dyslipidemia and arterial hypertension) and secondary to consumption of medications (amiodarone, tamoxifen, corticosteroids, estrogen, nifedipine) to metabolic,

1. MD (Internal Medicine), DMCH, Dhaka.

2. Associate Professor of Hepatology, SSMC, Dhaka.

3. Associate Professor Department of Medicine, DMCH, Dhaka.

4. Assistant Professor, Department of Medicine, DMCH, Dhaka.

5. Professor, Department of Medicine, DMCH, Dhaka. congenital or acquired alterations, nutrition, surgical procedures, and other toxins ${ }^{2,11}$. NAFLD is a metabolic disorder originally assumed to be largely confined to residents of affluent, industrialized Western countries $^{3}$; however, obesity and insulin resistance, the common substrates of NAFLD are not restricted to the west, as witnessed by their increasingly universal distribution ${ }^{4}$.In particular, there has been an upsurge in obesity-related metabolic syndrome in the Asia-Pacific region, so that in countries such as China, Japan, Korea and probably India, rates of NAFLD are between $12 \%$ and $24 \%$ of the general population ${ }^{5,10}$. There are critical differences with respect to the extent of adiposity between Eastern and Western populations ${ }^{6}$. Studies on body fat have shown that Asians have higher percentage adiposity at a lower Body Mass Index (BMI) than Caucasians ${ }^{7}$. Therefore, the recommended BMI cutoff values for Asians for overweight are $23-25 \mathrm{~kg}$ / $\mathrm{m}^{2}$ and for obesity $>25 \mathrm{~kg} / \mathrm{m}^{2}$ according to the new BMI criteria for Asians by World Heath Organization ${ }^{5}$. It should be emphasized that the distribution of fat may be more important than the total adipose mass. Central obesity is a correlate of visceral adiposity and is more closely linked to insulin resistance, the central event in Non-Alcoholic Steatohepatitis (NASH) than is generalized obesity ${ }^{8}$. Fatty liver can occur in apparently 'lean' but centrally obese individuals and its prevalence rises with further degrees of 
obesity especially central obesity ${ }^{9}$. In a recent, large crosssectional study of Shanghai adults, the risk of hepatic steatosis increased exponentially with each addition of the components of the metabolic syndrome ${ }^{12,13}$. There are a number of other risk factors for NAFLD have been identified from Asian studies. These include advanced age, gender, lower education, physical inactivity, high fat intake, overeating, recent weight gain, expanding waistline, family history of obesity and/or diabetes, family history of fatty liver, AST, platelet count of $>160000 / \mathrm{mL}^{12,14,15}$.

\section{Materials and Method:}

Selection of patient and control: This observational case control study was carried out at Medicine Department, Dhaka Medical College Hospital, Dhaka, during the period of August, 2011 to June, 2012. Following a purposive sampling method groups of 200 study populations who fulfill the inclusion and exclusion criteria were enrolled in the study. Fatty liver can be defined by the presence of at least two of three abnormal findings on abdominal ultrasonography: diffusely increased echogenicity ('bright') of liver greater than kidney or spleen, vascular blurring, and deep attenuation. Fatty liver was classified into mild, moderate and severe forms and will be graded on a scale of 0 to $3(0=$ none, $1=$ mild, $2=$ moderate, $3=$ severe $)$. Mild was defined as a slight increase in liver echogenicity and the relative preservation of echoes from the walls of the portal vein. Moderate was defined as moderate loss of echoes from the walls of the portal vein, particularly from the peripheral branches, and moderately increased echoegenecity. Severe was defined as a greater reduction in beam penetration, loss of echoes from most of the portal vein wall and extensive abnormally bright echoes. ${ }^{21}$

Inclusion criteria were non-alcoholic patients with fatty liver on ultrasound (Case) and non-alcoholic healthy subject without fatty liver on ultrasound (Control) between the age 15 and 70 years, who wish to voluntarily participate in the study by providing informed consent and who have not received previous treatment for NAFLD.

Exclusion criteria were history of alcohol intake, Hepatitis B virus surface antigen and hepatitis $\mathrm{C}$ antibodies positivity, Subjects with co morbid condition such as CCF \& CKD; Subjects who do not provide informed written consent, pregnant women; History of taking drugs for prolong period (glucocorticoids, OCP, Phenytoin, thiazide diltiazem, tamoxifen, amiodarone, herbal drugs etc) and Known case of Wilson's disease, autoimmune hepatitis, hamochromatosis. Controls are selected from patient relatives, hospital staff, doctors and doctor's relatives from DMCH matched for age and gender.
Procedure: After taking brief history including alcohol intake, preliminary selection was done and the purpose of the study was explained in details to each subjects. They were advised to take unrestricted carbohydrate diet, to do normal physical activities and to avoid drugs that significantly interfere with blood glucose level (like glucocorticoids, oral contraceptives containing levonorgestrel or high-dose estrogen, phenytoin, high-dose thiazide diuretics, etc) for 3 days. Then they were requested to come after 3 days at morning following an overnight (8-14 hours) fasting. When the subjects reported, informed written consent was taken. Then collection of history and physical examination was carried out according to predesigned case record form including demographic details of age, gender, contact information and hospital registration number. Patients were asked about history of diabetes, hypertension or intake of oral hypoglycemics, insulin, anti-hypertensive, amiodarone, diltiazem or tamoxifen (common drugs that cause hepatitis).

Investigation: From both cases and control, $5 \mathrm{ml}$ of venous blood was taken \& sent to pathology for CBC, fasting blood sugar, fasting lipid profile and liver function test. From cases S. creatinine, S. albumin, thyroid function test, HBSAg, anti HBCAg total, anti HCV, S. ferritin, 24 hours urinary copper, Anti Nuclear Antibody was sent when applicable. On the same visit their weight, height and waist circumference was measured and their Body Mass Index (BMI) was calculated.

Liver biopsy: After the investigation patients with one or more risk factors or increased transminases $>60 \mathrm{U} / 1$ was explained about the disease and its consequences and the importance of liver biopsy, its technique and possible complications. Those who gave consent to liver biopsy have their blood samples sent for complete blood picture, blood grouping, coagulation profile, and liver biopsy was taken using the trucut liver biopsy needle. The samples were sent to the pathology for report. Then NASH activity scoring had done to see the extent of liver damage. Statistical analysis: The data was entered and analyzed by using SPSS version 16.0 (Inc. Chicago III) software. Univariate, bivariate and multiple logistic regression analysis were performed, analyzing the qualitative variables with the chi-square test and the quantitative variables with the students-t test. Statistical significance was established at 5\% for all the analyses. Multivariate analysis was performed by means of a logistic regression model to observe the association of hypothetical risk factors of NAFLD by calculating the odds ratio for each of these factors. 


\section{Observations and Results:}

Majority of population belonged to $>40$ years in both groups. Male female ratio was $0.6: 1$. Brisk walking $<30$ minutes per day was found in $88 \%$ in NAFLD and $68 \%$ in control which was statistically significant $(\mathrm{P}<0.05)$. Mean waist circumference was found in $87.84 \pm 9.74 \mathrm{~cm}$ in NAFLD and $79.58 \pm 5.60 \mathrm{~cm}$ in control. The mean difference was statistically significant $(\mathrm{P}<0.05)$. Mean BMI was found $26.9 \pm 2.9 \mathrm{~kg} / \mathrm{m}^{2}$ and $25.7 \pm 3.6$ $\mathrm{kg} / \mathrm{m}^{2}$ in NAFLD and control group respectively and maximum $58 \%$ population were obese $(\mathrm{BMI}>25)$ in NAFLD which was statistically significant $(\mathrm{P}<0.05)$. The mean systolic $\mathrm{BP}$ was found $133.94 \pm 9.74 \mathrm{mmHg}$ in NAFLD and $129.8 \pm 11.5 \mathrm{mmHg}$ in control. The mean diastolic BP was found $83.45 \pm 5.76 \mathrm{mmHg}$ and $79.5 \pm 4.91 \mathrm{mmHg}$ in NAFLD and control respectively. The difference was statistically significant $(\mathrm{P}<0.05)$.
The mean fasting blood sugar was found $7.15 \pm 2.7 \mathrm{mmol} / \mathrm{l}$ in NAFLD and $5.9 \pm 1.6 \mathrm{mmol} / \mathrm{l}$ in control. Regarding the fasting lipid profile, the mean TG was found $243.0 \pm 114.0 \mathrm{mg} / \mathrm{dl}$ in NAFLD and $130.8 \pm 48.6 \mathrm{mg} / \mathrm{dl}$ in control. The mean LDL was found $110.0 \pm 40.5 \mathrm{mg} / \mathrm{dl}$ and $82.9 \pm 32.2 \mathrm{mg} / \mathrm{dl}$ in NAFLD and control respectively.

The mean HDL was found $40.5 \pm 8.31 \mathrm{mg} / \mathrm{dl}$ in NAFLD and $46.1 \pm 9.2 \mathrm{mg} / \mathrm{dl}$ in control. The mean TC was found $219.0 \pm 35.4$ $\mathrm{mg} / \mathrm{dl}$ and $215.0 \pm 28.5 \mathrm{mg} / \mathrm{dl}$ in NAFLD and control respectively. The mean TG, LDL, HDL and TC difference was statistically significant $(\mathrm{P}<0.05)$. Figure 1 showing metabolic syndrome, which was found $64 \%$ in NAFLD and $22 \%$ in non-NAFLD group which was significant $(<0.05 \%)$. Mild fatty changes were found $95 \%$ and moderate changes in $5 \%$ NAFLD patients.

Table-I

Distribution of the study population according to Physical activity, waist circumference, BMI and Blood pressure Fasting blood sugar and platelet count $(n=200)$.

\begin{tabular}{|c|c|c|c|c|c|}
\hline \multirow[t]{2}{*}{ Variables } & \multicolumn{2}{|c|}{ Case $(n=100)$} & \multicolumn{2}{|c|}{ Control $(n=100) P$ value } & \\
\hline & $\mathrm{n}$ & $\%$ & $\mathrm{n}$ & $\%$ & \\
\hline \multicolumn{6}{|l|}{ Physical activity } \\
\hline \multirow[t]{2}{*}{ Brisk walking $<30$ minutes per day } & 88 & 88.0 & 68 & 68.0 & $0.001^{\mathrm{s}}$ \\
\hline & Mean & $\pm \mathrm{SD}$ & Mean & $\pm \mathrm{SD}$ & \\
\hline Waist circumference (cm) & 87.84 & \pm 9.74 & 79.58 & \pm 5.60 & $0.001^{\mathrm{s}}$ \\
\hline Range (min-max) & $(34$ & $-112)$ & $(35$ & $-109)$ & \\
\hline $\operatorname{BMI}\left(\mathrm{kg} / \mathrm{m}^{2}\right)$ & 26.9 & \pm 2.9 & 25.7 & \pm 3.6 & $0.010^{\mathrm{s}}$ \\
\hline Range (min-max) & $(20.0$ & $-32.8)$ & $(20.0$ & $-31.8)$ & \\
\hline Systolic BP (mmHg) & 133.94 & \pm 9.74 & 129.8 & \pm 11.5 & $0.006^{\mathrm{s}}$ \\
\hline Range (min-max) & $(110$ & $-150)$ & $(115$ & $-145)$ & \\
\hline Diastolic BP (mmHg) & 83.45 & \pm 5.76 & 79.5 & \pm 4.91 & $0.001^{\mathrm{s}}$ \\
\hline Range (min-max) & $(70$ & $-95)$ & $(65$ & $-90)$ & \\
\hline Fasting Blood Sugar & 7.15 & \pm 2.7 & 5.9 & \pm 1.6 & $0.001^{\mathrm{s}}$ \\
\hline Range (min-max) & $(3.9$ & $-26.9)$ & $(4.8$ & $-25.0)$ & \\
\hline Platelet count (number $/ \mathrm{mm}^{3}$ ) & $2,36,151$ & \pm 73753 & $2,25,061$ & \pm 65846 & $0.263^{\mathrm{ns}}$ \\
\hline Range (min-max) & $(1,20,000$ & $-5,09,000)$ & $(1,15,000$ & $-5,08,000)$ & \\
\hline
\end{tabular}

Table-II

Distribution of the study population according to fasting lipid profile $(n=200)$.

\begin{tabular}{lccc}
\hline Variables & Case $(\mathrm{n}=100)$ & Control $(\mathrm{n}=100)$ & P Value \\
& Mean \pm SD & Mean \pm SD & $0.001^{\mathrm{s}}$ \\
\hline TG(mg/dl) & $243 \pm 114$ & $130.8 \pm 48.6$ & $(46-220)$ \\
Range (min-max) & $(68-456)$ & $82.9 \pm 32.2$ & $0.001^{\mathrm{s}}$ \\
LDL (mg/dl) & $110 \pm 40.5$ & $(34-148)$ & $0.001^{\mathrm{s}}$ \\
Range (min-max) & $(38-185)$ & $46.1 \pm 9.2$ & $0.029^{\mathrm{s}}$ \\
HDL (mg/dl) & $40.5 \pm 8.31$ & $(21-59)$ & \\
Range(min-max) & $(23-62)$ & $210.0 \pm 20.5$ & $(140-450)$ \\
TC (mg/dl) & $219.0 \pm 35.4$ & $(145-480)$ & \\
Range(min-max) & & & \\
\hline
\end{tabular}


Table-III

Binary logistic regression considering NAFLD as dependent variable and waist circumference, brisk walking, hypertension, diabetes, hypertriglyceridemia and hypercholesterolemia as independent variables $(n=200)$.

\begin{tabular}{lccccc}
\hline Independent variable & Bcoefficient & OR & \multicolumn{2}{c}{$95.0 \%$ CI for OR } & P value \\
\cline { 5 - 6 } & & & Lower & Upper & \\
\hline Waist circumference (> 90 cm in male and & 3.930 & 4.2 & 2.15 & 8.24 & $0.001^{\mathrm{s}}$ \\
$>80 \mathrm{~cm}$ in female) & & & & & \\
Brisk walking (<30 minutes/day) & 0.785 & 0.82 & 0.17 & 1.69 & $0.969^{\mathrm{ns}}$ \\
Hypertension & 1.548 & 0.33 & 0.14 & 1.11 & $0.826^{\mathrm{ns}}$ \\
Diabetes & -8.617 & 1.53 & 2.09 & 14.30 & $0.002^{\mathrm{s}}$ \\
Hypertriglyceridemia & 9.732 & 3.12 & 1.18 & 8.27 & $0.022^{\mathrm{s}}$ \\
Hypercholesterolemia & 0.616 & 0.72 & 0.12 & 1.47 & $0.993^{\mathrm{ns}}$ \\
\hline
\end{tabular}

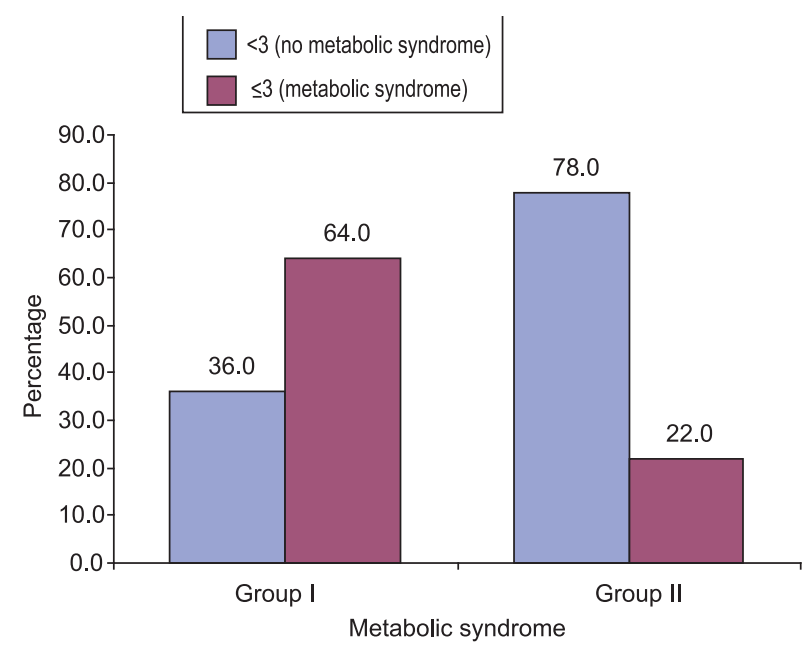

Fig-1: Bar diagram showing metabolic syndrome of the study patients.

After liver biopsy and histopathological findings NASH activity scoring was done to see the extent of liver damage. No definite NASH (score 0-5) was found 11(55.0\%) and definite NASH (score $>5$ ) was found $9(45.0 \%$ ) in study cases.

\section{Discussion:}

Non-alcoholic fatty liver disease (NAFLD) is becoming a public health problem with increasing incidence and it has been shown to be associated with T2DM, dyslipidemia, obesity and metabolic syndrome.

In this present series it was observed that the mean BMI was found $26.9 \pm 2.9 \mathrm{~kg} / \mathrm{m}^{2}$ and $25.7 \pm 3.6 \mathrm{~kg} / \mathrm{m}^{2}$ in NAFLD and non-NAFLD group respectively and maximum 58\% population were obese in NAFLD and $70 \%$ population were normal body weight in non-NAFLD group which was significantly $(\mathrm{P}<0.05)$ higher in NAFLD. Pagano et al. (2002) observed the mean BMI was found $26.0 \pm 2.0 \mathrm{~kg} / \mathrm{m}^{2}$ in NASH and $25.0 \pm 3.0 \mathrm{~kg} / \mathrm{m}^{2}$ in control group ${ }^{17}$.

The mean fasting blood sugar was found $7.15 \pm 2.7 \mathrm{mmol} / \mathrm{l}$ in NAFLD and $5.9 \pm 1.6 \mathrm{mmol} / \mathrm{l}$ in non-NAFLD group. Fasting blood sugar $>6.1 \mathrm{mmol} / 1$ were found in $57 \%$ and $>7.0 \mathrm{mmol} / 1$ were found in $38 \%$ cases which were significantly higher in NAFLD group. As a part of metabolic syndrome, the mean fasting blood sugar was significantly $(\mathrm{P}<0.05)$ higher in NAFLD group. In logistic regression analysis also showed that high FBS was independently associated with NAFLD group. Brea et al. (2005) found mean Fasting glucose, 6.4 \pm 1.3 $\mathrm{mmol} / \mathrm{L}$ in patients and $5.5 \pm 0.7 \mathrm{mmol} / \mathrm{L}$ in control ${ }^{19}$. Park et al. (2006) found raised fasting blood glucose 39.1\%in male and $34.6 \%$ in female, which are comparable with the current study $^{10}$.

Metabolic syndrome was found $64.0 \%$ in NAFLD and $22.0 \%$ in non-NAFLD group, which was significantly $(\mathrm{P}<0.05)$ higher in NAFLD. Marchesini et al. (2003) showed $88 \%$ of their study patents had a metabolic syndrome ${ }^{13}$. After liver biopsy and histopathological findings NASH activity scoring was done to see the extent of liver damage.

According to NASH Activity Scoring system no definite NASH (score 0-5) was found 11(55.0\%) and definite NASH (score $>5$ ) was found in $9(45.0 \%$ ) of the NAFLD group. So there is $45 \%$ of the ultrasonographically proved fatty liver patients who are developed NASH which is alarming and they have potential for the development of advanced liver disease.

The mean age was found $38.86 \pm 10.44$ years in NAFLD and $32.75 \pm 12.7$ years in non-NAFLD group with range from 18 to 58 years. The mean age was significantly $(\mathrm{P}<0.05)$ higher in NAFLD group. Majority of population were found belonged 
to $>40$ years in both groups. Probably aged populations were more prone to fatty liver due to more presence of metabolic syndrome. Recent study shown that, the mean age of the patients having NASH was $38 \pm 10$ years and $37 \pm 9$ years in control subjects, which is consistent with the current study ${ }^{17}$. Male were found $40.0 \%$ in NAFLD and $46.0 \%$ in non-NAFLD group. Male to female ratio was 1:1.3. Study result found male $63.3 \%$ and female $36.7 \%{ }^{11}$. Brisk walking Â30 minutes per day were $88.0 \%$ and $68.0 \%$ in NAFLD and non-NAFLD group respectively. It was significantly $(\mathrm{P}<0.05)$ higher in NAFLD group probably because those who walk less ( $<30$ minutes) is more likely to develop fatty liver. Hepatomegaly was found $44.0 \%$ and splenomegaly was found $6.0 \%$ in NAFLD which may be due to development of portal hypertension from cirrhosis of liver. A recent study showed $19.0 \%$ patients had hepatomegaly ${ }^{18}$. Mean systolic $\mathrm{BP}$ was found $133.94 \pm 9.74 \mathrm{mmHg}$ in NAFLD and $129.8 \pm 11.5$ $\mathrm{mmHg}$ in non-NAFLD group. The mean diastolic BP was found $83.45 \pm 5.76 \mathrm{mmHg}$ and $79.5 \pm 4.91 \mathrm{mmHg}$ in NAFLD and non-NAFLD group respectively that were significantly $(\mathrm{P}<0.05)$ higher in NAFLD. But logistic regression analysis showed that high systolic and diastolic BP was not associated with NAFLD. Pagano et al. (2002) observed that the mean systolic blood pressure was $132 \pm 14 \mathrm{mmHg}$ in NASH and $129 \pm 11 \mathrm{mmHg}$ in control group. Similarly, the mean diastolic blood pressure ( $\mathrm{mmHg}$ ) $90 \pm 9 \mathrm{mmHg}$ in NASH and $78 \pm 6 \mathrm{mmHg}$ in control group, which is closely resembled with the current study. ${ }^{17}$

Regarding the fasting lipid profile, in this study it was observed that the mean TG was found $243.0 \pm 114.0 \mathrm{mg} / \mathrm{dl}$ in NAFLD and $130.8 \pm 48.6 \mathrm{mg} / \mathrm{dl}$ in non-NAFLD group. The mean TC was found $219.0 \pm 35.4 \mathrm{mg} / \mathrm{dl}$ and $210.0 \pm 20.5 \mathrm{mg} / \mathrm{dl}$ in NAFLD and non-NAFLD group respectively. In logistic regression analysis showed that high TG was independently associated with NAFLD. Pagano et al. (2002) showed the mean triglycerides was $99 \pm 43 \mathrm{mg} / \mathrm{dL}$ in NASH and $75 \pm 31$ $\mathrm{mg} / \mathrm{dL}$ in control, mean total cholesterol was $175 \pm 36 \mathrm{mg} / \mathrm{dL}$ in NASH and $185 \pm 27 \mathrm{mg} / \mathrm{dL}$ in control ${ }^{17}$.

\section{Conclusion:}

NAFLD is an increasingly important chronic liver disease associated with high prevalence of more waist circumference, high FBS and high TG level. According to NAS scoring system definite NASH (score $>5$ ) was found $9(45.0 \%$ ) in NAFLD group which is alarming and they have potential for the development of advanced liver disease. So recognition of these risk factors in patients of NAFLD can help in early implementation of strategies that halt the progression of this disease. Future work is required to better define its natural history, elucidate the pathogenesis and develop effective treatment options for this progressive disease

\section{Conflict of Interest: None.}

\section{Reference:}

1. Ludwig, J, Viggiano, TR, McGill, DB, Oh, BJ. 'Non-alcoholic steatohepatitis. Mayo Clinic experiences with a hitherto unnamed disease' Mayo Clinic Proc. 1980; 55 (7): 434-438.

2. Bedogni, G, Bellentani, S. 'Fatty liver: How frequent is it and why', Ann Hepatol 2004; 3: 63-65.

3. Chitturi, S, Farrell, GC. 'Etiopathogenesis of non-alcoholic steatohepatitis’, Semin Liver Dis 2001; 21: 27-41.

4. Angulo, P. 'Non-alcoholic fatty liver disease', N Engl J Med 2002; 346: 1221-1331.

5. Williams, R. 'Global challenges in liver disease', Hepatology 2006; 44: 521-526.

6. Galassi, A, Reynolds, K, He, J. 'Metabolic syndrome and risk of cardiovascular disease: a meta-analysis', Am. J. Med 2006; 119: 812-19.

7. Wu, YF. 'Overweight and obesity in China. The once lean giant has a weight problem that is increasing rapidly', BMJ 2006; 19: 362-3.

8. Deurenberg, P, Deurenberg-Yap, M, Guricci, S. 'Asians are different from Caucasians and from each other in their body mass index/body fat per cent relationship', Obes. Rev 2002; 3: $141-6$.

9. Omagari, K, Kadokawa, Y, Masuda, J. 'Fatty liver in nonalcoholic non-overweight Japanese adults: incidence and clinical characteristics', J. Gastroenterol. Hepatol 2002; 17: 1098-105.

10. Hsieh, SD, Yoshinaga, H, Muto, T, Sakurai, Y, Kossaka, K. 'Health risks among Japanese men with moderate body index', Int. J. Obes. Relat, Metab. Disord 2000; 24: 358-62.

11. Park, HS, Han, JH, Lee, SY, Kim, DJ, Kim, SE. 'Prevalence of the metabolic syndrome among Korean adults according to the criteria of the International Diabetes Federation', Diabetes Care 2006; 29: 933-4.

12. Petersen, KF, Dufour, S, Feng, J. 'Increased prevalence of insulin resistance and non-alcoholic fatty liver disease in Asian-Indian men', Proc. Natl Acad. Sci USA 2006; 103: 18273-7.

13. Fan, JG, Zhu, J, Li, XJ. 'Fatty liver and the metabolic syndrome among Shanghai adults', J. Gastroenterol. Hepatol 2005; 20: 1825-32.

14. Marchesini, G, Bugianesi, E, Forlani, G. 'Non-alcoholic fatty liver, steatohepatitis, and the metabolic syndrome', Hepatology 2003; 37: 917-23.

15. Malik, A, Cheah, PL, Hilmi, IN, Chan, SP, Goh, KL. 'Nonalcoholic fatty liver disease in Malaysia: a demographic, 
anthropometric, metabolic and histological study', Chin. J. Dig. Dis 2007; 8: 58-64.

16. Adams, LA, Lymp, JF, St Sauver, J. 'The natural history of non-alcoholic fatty liver disease: a population-based cohort study', Gastroenterology 2005; 129: 113-21.

17. Chan, HLY, de Silva, HJ, Leung, NWY. 'How should we manage patients with non-alcoholic fatty liver disease in 2007', J. Gastroenterol. Hepatol 2007; 22: 801-8.

18. Pagano, C, Pacini, G, Musso, G, Gambino, R, Mecca, F, Depetris, N, Cassader, M, David, E, Cavallo-Perin, P, Rizzetto, M. 'Non-alcoholic steatohepatitis, insulin resistance and metabolic syndrome. Further evidence for an etiologic association', Hepatology 2002; 35: 367-72.
19. Bano, U, Gondal, M, Moin, S. 'Evaluation of risk factors of non- alcoholic fatty liver disease (nafld) in a tertiary care hospital at Rawalpindi, Pakistan: a local experience', JPMI 2008; 22(3): 189-195.

20. Brea, A, Mosquera, D, Martin, E, Arizti, A, Cordero, JL, Ros, E et al. 'Non-alcoholic fatty liver disease is associated with carotid atherosclerosis: a case-control study', Arterioscler. Thromb. Vasc. Biol 2005; 25: 1045-50.

21. Yajima, Y, Ohta, K, Narui, T, Abe, R, Suzuki, H, Ohtsuki, M. 'Ultrasonographical diagnosis of fatty liver: significance of the liver-kidney contrast', Tohoku J. Exp. Med, 1983; 139: 43-50. 